\title{
Does Acoustic Overlay of Music Improve Aerosol Penetration into Maxillary Sinuses?
}

\author{
Laurent Navarro*, Lara Leclerc and Jérémie Pourchez \\ University of Jean Monnet, France
}

*Corresponding author: Laurent Navarro, Univ Jean Monnet, 1 Mines Saint-Etienne, INSERM, U 1059 Sainbiose, Centre CIS, 158 cours Fauriel, F-42023 Saint-Etienne, France.

\author{
Received Date: June 04, 2019 \\ Published Date: June 17, 2019
}

\section{Mini Review}

Chronic rhinosinusitis (CRS) is a common disorder characterized by mucosal inflammation of the nose and paranasal sinuses. Recent years have seen numbers of findings and improvements in CRS treatment using nebulization process. Targeting delivery of nebulized antibiotics into the maxillary sinuses, the sites of infection, could improve clinical outcomes in patients with CRS. Thus, nasal drug delivery by nebulization is widely used in sinus disorders, because of its safety and convenience and due to its advantages as a painless therapy. However, the nebulization conditions to facilitate penetration of aerosols into the sinus cavities are not well established. The practice of aerosol therapy to treat CRS has not been studied thoroughly, despite few works have shown clinical benefit. This paper demonstrates that the music signal superimposed to aerosol provides a considerable innovative solution for the treatment of CRS patients. It encompasses frequency values among which the resonance frequency of the maxillary sinuses of patients regardless the initial sinus anatomy of the patient and the pathology stage and is thus more efficient to deliver the aerosol into the maxillary sinuses.

"Music heals". This adage is generally studied by neuropsychologists and music therapists based on psychological approaches [1]. Unfortunately, one knows that it is difficult to prove since it is hardly measurable [2]. Moreover, in these studies, music is used traditionally through speakers or headphones as it was designed for. In the meantime, it is well known that music has complex features in terms of signal properties, especially if regarded as a random signal. In short, music belongs to the class of pseudostationarity signals which implies some statistical randomness but also some stationarity. In addition, music has a large frequency spectrum which is mainly linked to the human ear's response of $20 \mathrm{~Hz}$ to $20 \mathrm{kHz}$. Most of the musical information lies in the $40 \mathrm{~Hz}-$ $16 \mathrm{kHz}$ range, and this range depends a lot on the musical style. These characteristics can be exploited when such frequency ranges must be used for medical treatments like nebulization process. Instead of using ear-fatiguing signals like sinusoidal, or sum of sinusoidal, signals. Then, music can be used for its characteristics as a signal. Patients are more likely to accept the presence and the hearing of a signal if it is music.

Chronic rhinosinusitis (CRS) is a disorder characterized by mucosal inflammation of the nose and paranasal sinuses. This common disease is marked by chronic sinonasal symptoms persisting for greater than 12 weeks that above all diminish patients' quality of life. It is a significant and increasing health problem which results in a large financial burden on society. CRS reportedly affects $5 \%$ to $13 \%$ of the general population in the United States, Europe and China [3]. Recent years have seen numbers of findings and improvements in CRS treatment using nebulization process [4-5]. Targeting delivery of nebulized antibiotics into the maxillary sinuses, the sites of infection, could improve clinical outcomes in patients with CRS. Thus, nasal drug delivery by nebulization is widely used in sinus disorders, because of its safety and convenience and due to its advantages as a painless therapy. Topical delivery of antimicrobial drugs for treatment of CRS also brings intuitive advantages over systemic therapy. It minimizes the risk of systemic side effects, the development of antibiotic resistance in non-targeted areas and allows a high topical drug concentration deposition with a minimal systemic adsorption.

However, the nebulization conditions to facilitate penetration of aerosols into the sinus cavities are not well-established. The practice of aerosol therapy to treat CRS has not been studied thoroughly, despite few works have shown clinical benefit. To enhance the penetration of nebulized particles into badly ventilated areas (i.e. sinuses in healthy subject) or non-ventilated areas (i.e. sinuses in patients with sinus diseases), a pressure gradient generated by an acoustic airflow can be added to a usual nebulizer. The effectiveness of the nebulization process is important because 
of the huge incidence of CRS, and the cost of the devices used in the process. This implies public authorities' awareness of the problem of efficiency and reimbursement of treatment.

Since the 1950s, acoustic airflow superimposed to nebulization process is empirically used as a medical treatment to improve the penetration of aerosols in nasal cavities mainly for the treatment of chronic rhinosinusitis. For long, the principle of sound superimposed to nebulization has been used with more or less success, using a sound which is basically a $100 \mathrm{~Hz}$ or $50 \mathrm{~Hz}$ sinusoidal signal. Historically, this value was found accidentally because of dust found in the sinuses of workers using electrical rotating machines inducing this type of sinusoidal signal. But today, this process is questioned by numerous studies and experiments. The hypothesis to explain this phenomenon was based on the Helmholtz resonator principle. In fact, the sinuses can be seen as cavities opened on a small tube with a relatively constant diameter.

As seductive as it may be, the Helmholtz resonator formula giving the fundamental frequency (Figure 1) is based on few elementary parameters. This simple empirical equation means that in good conditions of sphere volume (i.e. the maxillary sinus cavity in our case), length and diameter of the tube (i.e. the maxillary ostium in our case), one can find a resonance frequency of $100 \mathrm{~Hz}$. However, any geometric variation from this fragile equilibrium can lead to a wide range of different resonance frequencies, which means that using a fixed frequency of $100 \mathrm{~Hz}$ may be ideal only for very few patients. Looking closely to Figure 1, one can see that the inter-individual anatomical variability existing between patients can lead to large differences in the resulting resonant frequency. Moreover, even for a same patient with a given geometric features of maxillary sinuses, this resonant frequency can change during the evolution of the CRS. The inflammation and the mucus secretion can decrease the ostium diameter which is more or less obstructed, and the volume of the cavity can also vary due to the more or less fluid-filled sinus (Figure 1).

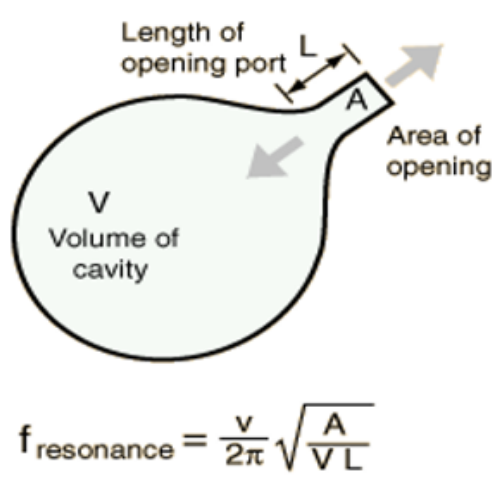

Figure 1: Helmholtz Resonator Formula Where $V$ is the Sound Speed in Air, And A, L and V Respectively the Section and the Length of the Tube, and the Volume of the Cavity.

In the past years, strategies have been developed to use varying acoustic frequencies that led to better results concerning penetration of aerosols in the sinuses [4-5]. These methods are based on the use of a frequency sweep that allow to reach all possible resonance frequencies within a specific range [5]. To allow for the choice of a wide variety of signals, it has been necessary to develop a specific device consisting of a membrane mounted on a vibrating pot linked to a computer through a power amplifier. This device led us to push the concept of using varying frequencies further while considering the patient's well-being. Considering that music is often associated with certain levels of well-being and enjoying the good properties of such signals, we decided to explore their efficiency for the use in the drug delivery process by nebulization to target the maxillary sinuses (Figure 2).

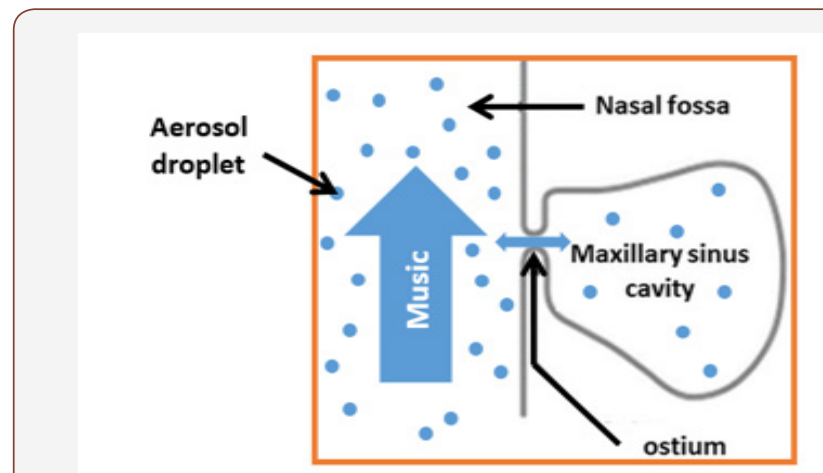

Figure 2: Principle of Music Aerosols. The Musical Signal Is Superimposed to The Aerosol to Improve the Penetration in the Maxillary Sinuses.

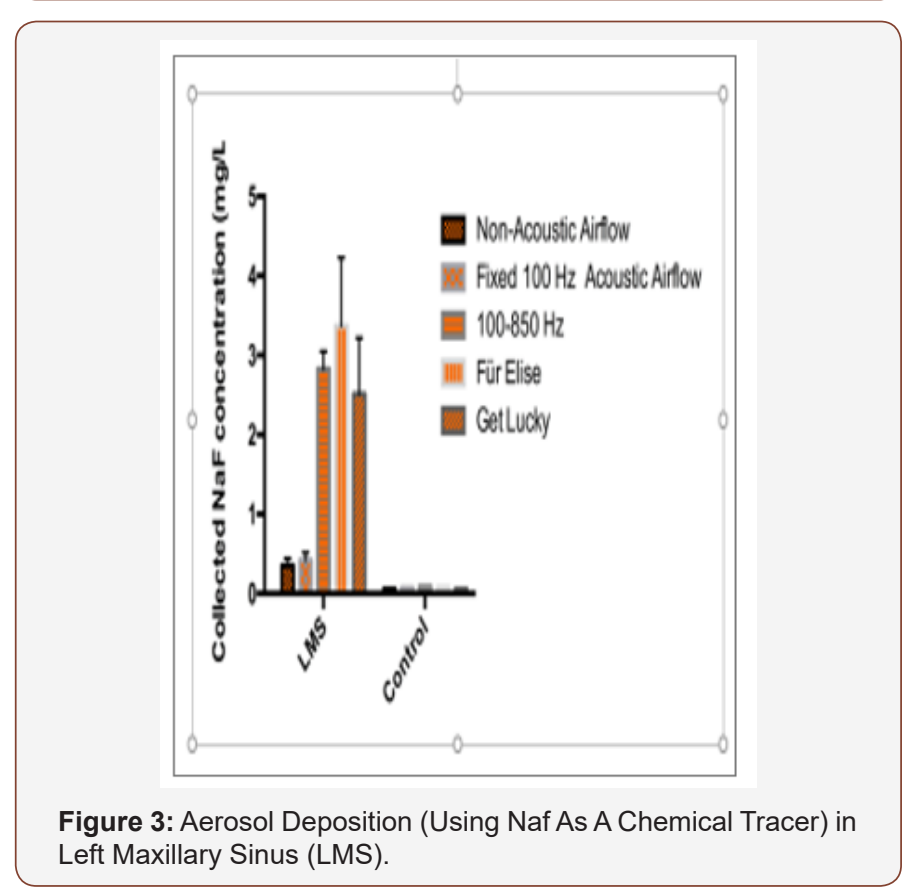

We tested two different musical pieces: "Für Elise" by Beethoven and "Get Lucky" by Daft Punk. The first one is categorized as classical music and is a quite smooth signal with no big amount of low frequencies, but a more complex behavior and few stationarities. The second one can be categorized in popular electronic music and is opposite to the first one with higher level of low frequencies and more stationarity due to the electronic kick beating approximatively two times per second. Using rigorously the same experimental procedure described in [5], the results we achieved exceeded what we expected. The first finding was that 
musical signals were clearly the best signals for aerosols deposition in sinuses, even at relatively low levels, compared to fixed $100 \mathrm{~Hz}$ frequency and acoustic sweep frequencies. To do so, we measured in a nasal replica the Sodium Fluoride $(\mathrm{NaF})$ deposition in maxillary sinuses, and we found that music and random noise of frequency range [100-850] were way more efficient to help the aerosol penetrate the sinuses. We measured ten times increase in the deposition for an amplitude of the signal of $80 \mathrm{~dB}$.

(Figure 3) Aerosol deposition (using NaF as a chemical tracer) in left maxillary sinus (LMS) for 5 different acoustic configurations: Non-acoustic airflow, Fixed $100 \mathrm{~Hz}$ sinusoidal acoustic airflow, 100-850 Hz random noise, "Für Elise" and "Get Lucky". Control experiments refers to nebulization of water without $\mathrm{NaF}$ chemical tracer. Experimental procedures described in [5].

In the light of results obtained in this study, the music signal superimposed to aerosol provides a considerable innovative solution for the treatment of CRS patients as it encompasses frequency values among which the resonance frequency of the maxillary sinuses of patients regardless the initial sinus anatomy of the patient and the pathology stage. Besides, the music aerosol is also patients-driven to make the aerosol treatment more funny and let them feel more comfortable in order to improve the patient compliance with airborne drug treatment.

\section{Acknowledgement}

None.

\section{Conflict of Interest}

No conflict of interest.

\section{References}

1. Kemper KJ, Danhauer SC (2005) Music as therapy. South Med J 98(3): 282-288.

2. Evans D (2002) The effectiveness of music as an intervention for hospital patients: a systematic review. J Adv Nurs 37(1): 8-18.

3. Agache I, Akdis C, Akdis M, Angier E, Arshad S, et al. (2015) Global atlas of allergic rhinitis and chronic rhinosinusitis, $1^{\text {st }}$ (edn). European Academy of Allergy and Clinical Immunology pp: 307.

4. Moghadam SJ, Navarro L, Leclerc L, Hodin S, Pourchez J (2018) Toward smart Nebulization: Engineering acoustic airflow to penetrate maxillary sinuses in chronic rhinosinusitis. Int J Pharm 546(1-2): 188-193.

5. El Merhie A, Navarro L, Delavenne X, Leclerc L, Pourchez J (2016) A new strategy to improve drug delivery to the maxillary sinuses: the frequency sweeps acoustic airflow. Pharm Res 33(5): 1074-1084. 